\title{
Advice for COVID-19 vaccination: get some sleep
}

\author{
Jie Zhu ${ }^{1} \cdot$ Mengqi Zhang ${ }^{1} \cdot$ Larry D. Sanford ${ }^{2} \cdot$ Xiangdong Tang $^{1}$
}

Received: 26 December 2020 / Revised: 18 January 2021 / Accepted: 3 February 2021 / Published online: 17 February 2021

(C) The Author(s), under exclusive licence to Springer Nature Switzerland AG part of Springer Nature 2021

To the Editor,

The global coronavirus disease 2019 (COVID-19) epidemic is showing signs of resurgence even as the public is looking forward to receiving the promising vaccines that are becoming available. In this stressful time, scientists and public health leaders should be aware that sleep disturbances are associated with vulnerabilities to infectious diseases and may affect the efficacy of vaccines. Sleep can enhance antigen $(\mathrm{Ag})$-specific $\mathrm{T}$ helper $(\mathrm{Th})$ cell and antibody $(\mathrm{Ab})$ responses. More slow-wave sleep reportedly results in increased growth hormone and prolactin levels and decreased cortisol release, which facilitates the transfer of antigenic information from antigen-presenting cells (APCs) to Ag-specific Th cells and fosters adaptive immune responses [1]. Repeated sleep restriction leads to lower antibody responses to hepatitis B [2] and influenza vaccines [3]. Even attaining sufficient sleep after vaccination cannot reverse the weakening of vaccine immunity.

It is essential for World Health Organization guidance to highlight the importance of sleep in the ongoing largescale COVID-19 vaccination efforts across population segments. Jobs that involve shift work (e.g., medical staff, night driver, and police) inevitably lead to acute sleep deprivation. Personnel should be advised to avoid getting vaccinated on shift days. If vaccination is planned during hospitalization, the sleep duration of inpatients should be considered because the change of environment, worries about illness, and medical care

Xiangdong Tang

2372564613@qq.com

1 Sleep Medicine Center, Department of Respiratory and Critical Care Medicine, Mental Health Center, West China Hospital, Sichuan University, Chengdu, China

2 Sleep Research Laboratory, Center for Integrative Neuroscience and Inflammatory Diseases, Department of Pathology and Anatomy, Eastern Virginia Medical School, Norfolk, VA, USA associated activities may affect sleep amount and quality. These factors may also aggravate the symptoms of chronic insomnia. Inpatients with obvious sleep disturbances may consider rescheduling the vaccination. In people with obstructive sleep apnea, telemedicine, and non-contact sleep guidance can ensure effective treatment around the vaccination. Additionally, excessive worries about the pandemic, health conditions, and economic impact contribute to impaired sleep in the general population [4]. Active strategies such as establishing a sleep routine, ensuring a quiet and safe sleep environment, avoiding blue light exposure before bedtime, and limiting alcohol, caffeine, or large meals before bedtime can support better vaccination protection [5], thus leading to a more effective halt in the global spread of COVID-19.

Acknowledgments Nothing declared.

Authors' contributions Jie Zhu is the primary author of this letter. Xiangdong Tang generated the original concept. Mengqi Zhang revised this letter with Larry D. Sanford.

Funding The present work was supported by the Key Program of the National Natural Science Foundation of China (Grant No.81530002).

\section{Declarations}

Conflict of interest None reported.

Disclosure statement All authors have seen and approved the manuscript. The opinions expressed do not necessarily reflect the official policy or position, either expressed or implied, of West China Hospital, Sichuan University or Eastern Virginia Medical School.

\section{References}

1. Lange T, Dimitrov S, Bollinger T, Diekelmann S, Born J (2011) Sleep after vaccination boosts immunological memory. J Immunol 187(1):283-290 
2. Prather AA, Hall M, Fury JM, Ross DC, Muldoon MF, Cohen S, Marsland AL (2012) Sleep and antibody response to hepatitis B vaccination. Sleep 35(8):1063-1069

3. Spiegel K, Sheridan JF, Van Cauter E (2002) Effect of sleep deprivation on response to immunization. JAMA 288(12):1471-1472

4. Miller MA, Cappuccio FP (2020) A systematic review of COVID-19 and obstructive sleep apnoea. Sleep Med Rev 55:101382
5. American Academy of Sleep Medicine. About the National Healthy Sleep Awareness Project. http:// https://aasm.org/healthy-sleep-andimmune-response-to-covid-19-vaccination/. Accessed 13 Jan 2021

Publisher's note Springer Nature remains neutral with regard to jurisdictional claims in published maps and institutional affiliations. 